\title{
The Intersection of Fixed Point Subgroups by Involutive Automorphisms of Compact Exceptional Lie Groups
}

\author{
Toshikazu MIYASHITA
}

Nagano Prefectual Komoro Senior high School

(Communicated by Y. Maeda)

\begin{abstract}
In this paper we treat the intersection of fixed point subgroups by the involutive automorphisms of exceptional Lie group $G=F_{4}, E_{6}, E_{7}$. We shall find involutive automorphisms of $G$ such that the connected component of the intersection of those fixed point subgroups coincides with the maximal torus of $G$.
\end{abstract}

\section{Introduction}

It is known that the involutive automorphisms of the compact Lie groups play an important role in the theory of symmetric space (c.f. Berger [1]). In [8], [9] Yokota showed that the exceptional symmetric spaces $G / H$ are realized definitely by calculating the fixed point subgroup of the involutive automorphisms $\tilde{\gamma}, \tilde{\gamma}^{\prime}, \tilde{\sigma}, \tilde{\sigma}^{\prime}, \tilde{\iota}$ of $G$, where $\tilde{\gamma}, \tilde{\gamma}^{\prime}, \tilde{\sigma}, \tilde{\sigma}^{\prime}$ are induced by $\mathbf{R}$-linear transformations $\gamma, \gamma^{\prime}, \sigma, \sigma^{\prime}$ of $\mathfrak{J}$ and $\tilde{\imath}$ is induced by $C$-linear transformation $\iota$ of $\mathfrak{J}^{C}$. Here $\gamma, \gamma^{\prime} \in G_{2} \subset F_{4} \subset E_{6} \subset E_{7}$ and $\sigma, \sigma^{\prime} \in F_{4} \subset E_{6} \subset E_{7}$ and $\iota \in E_{7}$. For the cases of the graded Lie algebras $\mathfrak{g}$ of the second kind and third kind, the corresponding subalgebras $\mathfrak{g}_{0}, \mathfrak{g}_{e v}, \mathfrak{g}_{e d}$ of $\mathfrak{g}$ are realized as the intersection of those fixed point subgroups of the commutative involutive automorphisms ([3], [6], [7], [10], [11], [12]).

In [2], [4], [5] we determined the intersection of those fixed point subgroups of the involutive automorphisms of $G$ when $G$ is a compact exceptional Lie group. We remark that those intersection subgroups are maximal rank of $G$.

In general, let $G$ be a connected compact Lie group and $\sigma_{1}, \sigma_{2}, \ldots, \sigma_{m}$ commutative involutive elements of $G$. Set $G^{\sigma_{1}, \sigma_{2}, \ldots, \sigma_{k}}=\left\{\alpha \in G \mid \sigma_{i} \alpha=\alpha \sigma_{i}, i=1, \ldots, k\right\}$. We expect that the group $G^{\sigma_{1}, \sigma_{2}, \ldots, \sigma_{k}}$ is a maximal rank subgroup of $G$. Consider the following degreasing sequence of subgroups of $G$ :

$$
G^{\sigma_{1}} \supset G^{\sigma_{1}, \sigma_{2}} \supset \cdots \supset G^{\sigma_{1}, \ldots, \sigma_{m}} .
$$

Let $T^{l}$ be the maximal tours of $G$. In this paper we would like to find $\sigma_{1}, \sigma_{2}, \ldots, \sigma_{m}$ such that the connected component subgroup $\left(G^{\sigma_{1}, \sigma_{2}, \ldots, \sigma_{k}}\right)_{0}$ of the group $G^{\sigma_{1}, \sigma_{2}, \ldots, \sigma_{k}}$ is isomorphic to $T^{l}$ when $G$ is simply connected compact exceptional Lie groups $G_{2}, F_{4}, E_{6}$ or $E_{7}$. For the 
case $G=G_{2}$, we prove that the group $\left(\left(G_{2}\right)^{\gamma, \gamma^{\prime}}\right)_{0} \cong T^{2}$ by [5], Theorem 1.1.3. Then we shall prove the following:

$$
\begin{aligned}
& \text { (1) }\left(\left(F_{4}\right)^{\gamma, \gamma^{\prime}, \sigma, \sigma^{\prime}}\right)_{0} \cong T^{4}, \\
& \text { (2) }\left(\left(E_{6}\right)^{\gamma, \gamma^{\prime}, \sigma, \sigma^{\prime}}\right)_{0} \cong T^{6}, \\
& \text { (3) }\left(\left(E_{7}\right)^{\gamma, \gamma^{\prime}, \sigma, \sigma^{\prime}, \iota}\right)_{0} \cong T^{7} .
\end{aligned}
$$

For the case $G=E_{8}$, we conjecture that the group $\left(\left(E_{8}\right)^{\gamma, \gamma^{\prime}, \sigma, \sigma^{\prime}, v_{3}}\right)_{0} \cong T^{8}$, where $v_{3} \in E_{8}$ (As for $v_{3}$, see [3]).

\section{Group $F_{4}$}

The simply connected compact Lie group $F_{4}$ is given by the automorphism group of the exceptional Freudenthal algebra $\mathfrak{J}$ :

$$
F_{4}=\left\{\alpha \in \operatorname{Iso}_{\mathbf{R}}(\mathfrak{J}) \mid \alpha(X \times Y)=\alpha X \times \alpha Y\right\} .
$$

We shall review the definitions of $\mathbf{R}$-linear transformations $\gamma, \gamma^{\prime}, \sigma, \sigma^{\prime}$ of $\mathfrak{J}([8],[10]$, [12]).

Firstly we define $\mathbf{R}$-linear transformations $\gamma, \gamma^{\prime}$ and $\gamma_{1}$ of $\mathfrak{J}_{\mathbf{C}} \oplus M(3, \mathbf{C})=\mathfrak{J}$ by

$$
\begin{aligned}
& \gamma(X+M)=X+\gamma\left(\mathbf{m}_{1}, \mathbf{m}_{2}, \mathbf{m}_{3}\right)=X+\left(\gamma \mathbf{m}_{1}, \gamma \mathbf{m}_{2}, \gamma \mathbf{m}_{3}\right), \\
& \gamma^{\prime}(X+M)=X+\gamma^{\prime}\left(\mathbf{m}_{1}, \mathbf{m}_{2}, \mathbf{m}_{3}\right)=X+\left(\gamma^{\prime} \mathbf{m}_{1}, \gamma^{\prime} \mathbf{m}_{2}, \gamma^{\prime} \mathbf{m}_{3}\right), \\
& \gamma_{1}(X+M)=\bar{X}+\bar{M}, \quad X+M \in \mathfrak{J}_{\mathbf{C}} \oplus M(3, \mathbf{C})=\mathfrak{J},
\end{aligned}
$$

respectively, where $\mathfrak{J}_{\mathbf{C}}=\left\{X \in M(3, \mathbf{C}) \mid X^{*}=X\right\}$, the right-hand side transformations $\gamma, \gamma^{\prime}: \mathbf{C}^{3} \rightarrow \mathbf{C}^{3}$ are defined by

$$
\gamma\left(\left(\begin{array}{l}
n_{1} \\
n_{2} \\
n_{3}
\end{array}\right)\right)=\left(\begin{array}{c}
n_{1} \\
-n_{2} \\
-n_{3}
\end{array}\right), \quad \gamma^{\prime}\left(\left(\begin{array}{l}
n_{1} \\
n_{2} \\
n_{3}
\end{array}\right)\right)=\left(\begin{array}{c}
-n_{1} \\
n_{2} \\
-n_{3}
\end{array}\right), \quad n_{i} \in \mathbf{C}
$$

Then $\gamma, \gamma^{\prime}, \gamma_{1} \in G_{2} \subset F_{4}$, and $\gamma^{2}=\gamma^{\prime 2}=\gamma_{1}^{2}=1$.

Further we define $\mathbf{R}$-linear transfomations $\sigma$ and $\sigma^{\prime}$ of $\mathfrak{J}_{\mathbf{C}} \oplus M(3, \mathbf{C})=\mathfrak{J}$ by

$$
\begin{aligned}
\sigma(X+M) & =\sigma X+\left(\mathbf{m}_{1},-\mathbf{m}_{2},-\mathbf{m}_{3}\right), \\
\sigma^{\prime}(X+M) & =\sigma^{\prime} X+\left(-\mathbf{m}_{1},-\mathbf{m}_{2}, \mathbf{m}_{3}\right), \quad X+M \in \mathfrak{J}_{\mathbf{C}} \oplus M(3, \mathbf{C})=\mathfrak{J},
\end{aligned}
$$

respctively, where the right-hand side transformations $\sigma, \sigma^{\prime}: \mathfrak{J}_{\mathbf{C}} \rightarrow \mathfrak{J}_{\mathbf{C}}$ are defined by

$$
\sigma X=\sigma\left(\begin{array}{ccc}
\xi_{1} & x_{3} & \bar{x}_{2} \\
\bar{x}_{3} & \xi_{2} & x_{1} \\
x_{2} & \bar{x}_{1} & \xi_{3}
\end{array}\right)=\left(\begin{array}{ccc}
\xi_{1} & -x_{3} & -\bar{x}_{2} \\
-\bar{x}_{3} & \xi_{2} & x_{1} \\
-x_{2} & \bar{x}_{1} & \xi_{3}
\end{array}\right), \quad \sigma^{\prime} X=\left(\begin{array}{ccc}
\xi_{1} & x_{3} & -\bar{x}_{2} \\
\bar{x}_{3} & \xi_{2} & -x_{1} \\
-x_{2} & -\bar{x}_{1} & \xi_{3}
\end{array}\right)
$$


Then $\sigma, \sigma^{\prime} \in F_{4}$ and $\sigma^{2}=\sigma^{\prime 2}=1$.

The group $\mathbf{Z}_{2}=\left\{1, \gamma_{1}\right\}$ acts on the group $U(1) \times U(1) \times S U(3)$ by

$$
\gamma_{1}(p, q, A)=(\bar{p}, \bar{q}, \bar{A}) .
$$

Hence the group $\mathbf{Z}_{2}=\left\{1, \gamma_{1}\right\}$ acts naturally on the group $(U(1) \times U(1) \times S U(3)) / \mathbf{Z}_{3}$.

Let $(U(1) \times U(1) \times S U(3)) \cdot \mathbf{Z}_{2}$ be the semi-direct product of those groups under this action.

Hereafter, $\omega_{1}$ denotes $-\frac{1}{2}+\frac{\sqrt{3}}{2} e_{1} \in \mathfrak{C}$.

PROPOSITION 2.1. $\left(F_{4}\right)^{\gamma, \gamma^{\prime}} \cong\left((U(1) \times U(1) \times S U(3)) / \mathbf{Z}_{3}\right) \cdot \mathbf{Z}_{2}, \mathbf{Z}_{3}=\{(1,1, E)$, $\left.\left(\omega_{1}, \omega_{1}, \omega_{1} E\right),\left(\omega_{1}^{2}, \omega_{1}^{2}, \omega_{1}^{2} E\right)\right\}$.

PROOF. We define a mapping $\varphi_{4}:(U(1) \times U(1) \times S U(3)) \cdot \mathbf{Z}_{2} \rightarrow\left(F_{4}\right)^{\gamma, \gamma^{\prime}}$ by

$$
\begin{aligned}
& \varphi_{4}((p, q, A), 1)(X+M)=A X A^{*}+D(p, q) M A^{*}, \\
& \varphi_{4}\left((p, q, A), \gamma_{1}\right)(X+M)=A \bar{X} A^{*}+D(p, q) \bar{M} A^{*}, \\
& X+M \in \mathfrak{J}_{\mathbf{C}} \oplus M(3, \mathbf{C})=\mathfrak{J},
\end{aligned}
$$

where $D(p, q)=\operatorname{diag}(p, q, \bar{p} q) \in S U(3)$. Then $\varphi_{4}$ induces the required isomorphism (see [5] for details).

LEMMA 2.2. The mapping $\varphi_{4}:(U(1) \times U(1) \times S U(3)) \cdot \mathbf{Z}_{2} \rightarrow\left(F_{4}\right)^{\gamma, \gamma^{\prime}}$ satisfies

$$
\sigma=\varphi_{4}\left(\left(1,1, E_{1,-1}\right), 1\right), \quad \sigma^{\prime}=\varphi_{4}\left(\left(1,1, E_{-1,1}\right), 1\right),
$$

where $E_{1,-1}=\operatorname{diag}(1,-1,-1), E_{-1,1}=\operatorname{diag}(-1,-1,1) \in S U(3)$.

We denote $U(1) \times \cdots \times U(1),(1, \ldots, 1)$ and $\left(\omega_{k}, \ldots, \omega_{k}\right)(l$-times $)$ by $U(1)^{\times l},(1)^{\times l}$ and $\left(\omega_{k}\right)^{\times l}$, respectively.

Now, we determine the structures of the group $\left(F_{4}\right)^{\gamma, \gamma^{\prime}, \sigma, \sigma^{\prime}}=\left(\left(F_{4}\right)^{\gamma, \gamma^{\prime}}\right)^{\sigma, \sigma^{\prime}}$.

THEOREM 2.3. $\left(\left(F_{4}\right)^{\gamma, \gamma^{\prime}, \sigma, \sigma^{\prime}}\right)_{0} \cong U(1)^{\times 4}$.

PROOF. For $\alpha \in\left(F_{4}\right)^{\gamma, \gamma^{\prime}, \sigma, \sigma^{\prime}} \subset\left(F_{4}\right)^{\gamma, \gamma^{\prime}}$, there exist $p, q \in U(1)$ and $A \in S U(3)$ such that $\alpha=\varphi_{4}((p, q, A), 1)$ or $\alpha=\varphi_{4}\left((p, q, A), \gamma_{1}\right)$ (Proposition 2.1). For the case of $\alpha=\varphi_{4}((p, q, A), 1)$, by combining the conditions of $\sigma \alpha \sigma=\alpha$ and $\sigma^{\prime} \alpha \sigma^{\prime}=\alpha$ with Lemma 2.2, we have

$$
\varphi_{4}\left(\left(p, q, E_{1,-1} A E_{1,-1}\right), 1\right)=\varphi_{4}((p, q, A), 1)
$$

and

$$
\varphi_{4}\left(\left(p, q, E_{-1,1} A E_{-1,1}\right), 1\right)=\varphi_{4}((p, q, A), 1) .
$$


Hence
(i) $E_{1,-1} A E_{1,-1}=A$,
(ii) $\left\{\begin{array}{l}p=\omega_{1} p \\ q=\omega_{1} q \\ E_{1,-1} A E_{1,-1}=\omega_{1} A\end{array}\right.$
(iii) $\left\{\begin{array}{l}p=\omega_{1}^{2} p \\ q=\omega_{1}^{2} q \\ E_{1,-1} A E_{1,-1}=\omega_{1}{ }^{2} A\end{array}\right.$

and
(iv) $E_{-1,1} A E_{-1,1}=A$,
(v) $\left\{\begin{array}{l}p=\omega_{1} p \\ q=\omega_{1} q \\ E_{-1,1} A E_{-1,1}=\omega_{1} A\end{array}\right.$
(vi) $\left\{\begin{array}{l}p=\omega_{1}^{2} p \\ q=\omega_{1}^{2} q \\ E_{-1,1} A E_{-1,1}=\omega_{1}^{2} A .\end{array}\right.$

We can eliminate the case (ii), (iii), (v) or (vi) because $p \neq 0$ or $q \neq 0$. Hence we have $p, q \in U(1)$ and $A \in S(U(1) \times U(1) \times U(1))$. Since the mapping $U(1) \times U(1) \rightarrow S(U(1) \times$ $U(1) \times U(1))$,

$$
h\left(a_{1}, a_{2}\right)=\left(a_{1}, a_{2}, \overline{a_{1} a_{2}}\right)
$$

is an isomorphism, the group satisfying with the conditions of case (i) and (iv) is $\left(U(1)^{\times 4}\right) / \mathbf{Z}_{3}$. For the case of $\alpha=\varphi_{4}\left((p, q, A), \gamma_{1}\right)$, from $\varphi_{4}\left((p, q, A), \gamma_{1}\right)=\varphi_{4}((p, q, A)$, 1) $\gamma_{1}, \varphi_{4}\left(\left(1,1, E_{1,-1}\right), 1\right) \gamma_{1}=\gamma_{1} \varphi_{4}\left(\left(1,1, E_{1,-1}\right), 1\right)$ and $\varphi_{4}\left(\left(1,1, E_{-1,1}\right), 1\right) \gamma_{1}=\gamma_{1} \varphi_{4}((1$, $\left.\left.1, E_{-1,1}\right), 1\right)$, this case is in the same situation as above. Thus we have $\left(F_{4}\right)^{\gamma, \gamma^{\prime}, \sigma, \sigma^{\prime}} \cong$ $\left(\left(U(1)^{\times 4}\right) / \mathbf{Z}_{3}\right) \cdot \mathbf{Z}_{2}, \mathbf{Z}_{3}=\left\{(1)^{\times 4},\left(w_{1}\right)^{\times 4},\left(w_{1}^{2}\right)^{\times 4}\right\}$. The group $\left(U(1)^{\times 4}\right) / \mathbf{Z}_{3}$ is naturally isomorphic to the torus $U(1)^{\times 4}$, hence we obtain $\left(F_{4}\right)^{\gamma, \gamma^{\prime}, \sigma, \sigma^{\prime}} \cong\left(U(1)^{\times 4}\right) \cdot \mathbf{Z}_{2}$. Therefore we have the required isomorphism of the theorem.

\section{The group $E_{6}$}

The simply connected compact Lie group $E_{6}$ is given by

$$
E_{6}=\left\{\alpha \in \operatorname{Iso}_{C}\left(\mathfrak{J}^{C}\right) \mid \alpha X \times \alpha Y=\tau \alpha \tau(X \times Y),\langle\alpha X, \alpha Y\rangle=\langle X, Y\rangle\right\} .
$$

$\mathbf{R}$-linear transformations $\gamma, \gamma^{\prime}, \gamma_{1}, \sigma$ and $\sigma^{\prime}$ of $\mathfrak{J}=\mathfrak{J}_{\mathbf{C}} \oplus M(3, \mathbf{C})$ are naturally extended to the $C$-linear transformations of $\gamma, \gamma^{\prime}, \gamma_{1}, \sigma$ and $\sigma^{\prime}$ of $\mathfrak{J}^{C}=\left(\mathfrak{J}_{\mathbf{C}}\right)^{C} \oplus M(3, \mathbf{C})^{C}$. Then we have $\gamma, \gamma^{\prime}, \gamma_{1}, \sigma, \sigma^{\prime} \in E_{6}$.

The group $\mathbf{Z}_{2}=\left\{1, \gamma_{1}\right\}$ acts on the group $U(1) \times U(1) \times S U(3) \times S U(3)$ by

$$
\gamma_{1}(p, q, A, B)=(\bar{p}, \bar{q}, \bar{B}, \bar{A}) .
$$

Hence the group $\mathbf{Z}_{2}=\left\{1, \gamma_{1}\right\}$ acts naturally on the group $(U(1) \times U(1) \times S U(3) \times S U(3)) / \mathbf{Z}_{3}$.

Let $(U(1) \times U(1) \times S U(3) \times S U(3)) \cdot \mathbf{Z}_{2}$ be the semi-direct product of those groups under this action.

Proposition 3.1. $\left(E_{6}\right)^{\gamma, \gamma^{\prime}} \cong\left((U(1) \times U(1) \times S U(3) \times S U(3)) / \mathbf{Z}_{3}\right) \cdot \mathbf{Z}_{2}, \mathbf{Z}_{3}=$ $\left\{(1,1, E, E),\left(\omega_{1}, \omega_{1}, \omega_{1} E, \omega_{1} E\right),\left(\omega_{1}^{2}, \omega_{1}^{2}, \omega_{1}^{2} E, \omega_{1}^{2} E\right)\right\}$. 
Proof. We define a mapping $\varphi_{6}:(U(1) \times U(1) \times S U(3) \times S U(3)) \cdot \mathbf{Z}_{2} \rightarrow\left(E_{6}\right)^{\gamma, \gamma^{\prime}}$ by

$$
\begin{aligned}
& \varphi_{6}((p, q, A, B), 1)(X+M)=h(A, B) X h(A, B)^{*}+D(p, q) M \tau h(A, B)^{*}, \\
& \varphi_{6}\left((p, q, A, B), \gamma_{1}\right)(X+M)=h(A, B) \bar{X} h(A, B)^{*}+D(p, q) \bar{M} \tau h(A, B)^{*}, \\
& X+M \in\left(\mathfrak{J}_{\mathbf{C}}\right)^{C} \oplus M(3, \mathbf{C})^{C}=\mathfrak{J}^{C} .
\end{aligned}
$$

Here $D(p, q)=\operatorname{diag}(p, q, \overline{p q}) \in S U(3)$ and $h: M(3, \mathbf{C}) \times M(3, \mathbf{C}) \rightarrow M(6, \mathbf{C})^{C}$ is defined by

$$
h(A, B)=\frac{A+B}{2}+i \frac{A-B}{2} e_{1} .
$$

Then $\varphi_{6}$ induces the required isomorphism (see [5] for details).

LEMMA 3.2. The mapping $\varphi_{6}:(U(1) \times U(1) \times S U(3) \times S U(3)) \cdot \mathbf{Z}_{2} \rightarrow\left(E_{6}\right)^{\gamma, \gamma^{\prime}}$ satisfies

$$
\sigma=\varphi_{6}\left(\left(1,1, E_{1,-1}, E_{1,-1}\right), 1\right), \quad \sigma^{\prime}=\varphi_{6}\left(\left(1,1, E_{-1,1}, E_{-1,1}\right), 1\right)
$$

The group $\mathbf{Z}_{2}=\left\{1, \gamma_{1}\right\}$ acts on the group $U(1)^{\times 6}$ by

$$
\gamma_{1}\left(p, q, a_{1}, a_{2}, a_{3}, a_{4}\right)=\left(\bar{p}, \bar{q}, \bar{a}_{3}, \bar{a}_{4}, \bar{a}_{1}, \bar{a}_{2}\right) .
$$

Let $\left(U(1)^{\times 6}\right) \cdot \mathbf{Z}_{2}$ be the semi-direct product of those groups under this action.

Now, we determine the structures of the gruop $\left(E_{6}\right)^{\gamma, \gamma^{\prime}, \sigma, \sigma^{\prime}}=\left(\left(E_{6}\right)^{\gamma, \gamma^{\prime}}\right)^{\sigma, \sigma^{\prime}}$.

THEOREM 3.3. $\left(\left(E_{6}\right)^{\gamma, \gamma^{\prime}, \sigma, \sigma^{\prime}}\right)_{0} \cong U(1)^{\times 6}$.

PROOF. For $\alpha \in\left(E_{6}\right)^{\gamma, \gamma^{\prime}, \sigma, \sigma^{\prime}} \subset\left(E_{6}\right)^{\gamma, \gamma^{\prime}}$, there exist $p, q \in U(1)$ and $A, B \in S U(6)$ such that $\alpha=\varphi_{6}((p, q, A, B), 1)$ or $\alpha=\varphi_{6}\left((p, q, A, B), \gamma_{1}\right)$ (Proposition 3.1). For the case of $\alpha=\varphi_{6}((p, q, A, B), 1)$, by combining the conditions $\sigma \alpha \sigma=\alpha$ and $\sigma^{\prime} \alpha \sigma^{\prime}=\alpha$ with Lemma 3.2, we have

$$
\varphi_{6}\left(\left(p, q, E_{1,-1} A E_{1,-1}, E_{1,-1} B E_{1,-1}\right), 1\right)=\varphi_{6}((p, q, A, B), 1)
$$

and

$$
\varphi_{6}\left(\left(p, q, E_{-1,1} A E_{-1,1}, E_{-1,1} B E_{-1,1}\right), 1\right)=\varphi_{6}((p, q, A, B), 1) .
$$

Hence
(i) $\left\{\begin{array}{l}E_{1,-1} A E_{1,-1}=A \\ E_{1,-1} B E_{1,-1}=B,\end{array}\right.$
(ii) $\left\{\begin{array}{l}p=\omega_{1} p \\ q=\omega_{1} q \\ E_{1,-1} A E_{1,-1}=\omega_{1} A \\ E_{1,-1} B E_{1,-1}=\omega_{1} B\end{array}\right.$
(iii) $\left\{\begin{array}{l}p=\omega_{1}^{2} p \\ q=\omega_{1}^{2} q \\ E_{1,-1} A E_{1,-1}=\omega_{1}^{2} A \\ E_{1,-1} B E_{1,-1}=\omega_{1}^{2} B\end{array}\right.$ 
and

(iv) $\left\{\begin{array}{l}E_{-1,1} A E_{-1,1}=A \\ E_{-1,1} B E_{-1,1}=B\end{array}\right.$ (v) $\left\{\begin{array}{l}p=\omega_{1} p \\ q=\omega_{1} q \\ E_{-1,1} A E_{-1,1}=\omega_{1} A \\ E_{-1,1} B E_{-1,1}=\omega_{1} B\end{array} \quad\right.$ (vi) $\left\{\begin{array}{l}p=\omega_{1}^{2} p \\ q=\omega_{1}^{2} q \\ E_{-1,1} A E_{-1,1}=\omega_{1}^{2} A \\ E_{-1,1} B E_{-1,1}=\omega_{1}^{2} B .\end{array}\right.$

We can eliminate the case (ii), (iii), (v) or (vi) because $p \neq 0$ or $q \neq 0$. Thus we have $p, q \in U(1)$ and $A, B \in S\left(U(1)^{\times 3}\right)$. Since the mapping $U(1)^{\times 4} \rightarrow S\left(U(1)^{\times 5}\right)$,

$$
h\left(a_{1}, a_{2}, a_{3}, a_{4}\right)=\left(a_{1}, a_{2}, a_{3}, a_{4}, \overline{a_{1} a_{2} a_{3} a_{4}}\right)
$$

is an isomorphism, the group satisfying with the conditions of case (i) and (iv) is $\left(U(1)^{\times 6}\right) / \mathbf{Z}_{3}$. For the case of $\alpha=\varphi_{6}\left((p, q, A, B), \gamma_{1}\right)$, from $\varphi_{6}\left((p, q, A, B), \gamma_{1}\right)=$ $\varphi_{6}((p, q, A, B), 1) \gamma_{1}, \varphi_{6}\left(\left(1,1, E_{1,-1}, E_{1,-1}\right), 1\right) \gamma_{1}=\gamma_{1} \varphi_{6}\left(\left(1,1, E_{1,-1}, E_{1,-1}\right), 1\right)$ and $\varphi_{6}\left(\left(1,1, E_{-1,1}, E_{-1,1}\right), 1\right) \gamma_{1}=\gamma_{1} \varphi_{6}\left(\left(1,1, E_{-1,1}, E_{-1,1}\right), 1\right)$, this case is in the same situation as above. Thus we have $\left(E_{6}\right)^{\gamma, \gamma^{\prime}, \sigma, \sigma^{\prime}} \cong\left(\left(U(1)^{\times 6}\right) / \mathbf{Z}_{3}\right) \cdot \mathbf{Z}_{2}, \mathbf{Z}_{3}=\left\{(1)^{\times 6},\left(w_{1}\right)^{\times 6}\right.$, $\left(w_{1}^{2}\right)^{\times 6}$. The group $\left(U(1)^{\times 6}\right) / \mathbf{Z}_{3}$ is naturally isomorphic to the torus $U(1)^{\times 6}$, hence we obtain $\left(E_{6}\right)^{\gamma, \gamma^{\prime}, \sigma, \sigma^{\prime}} \cong\left(U(1)^{\times 6}\right) \cdot \mathbf{Z}_{2}$. Therefore we have the required isomorphism of the theorem.

\section{Group $E_{7}$}

Let $\mathfrak{P}^{C}=\mathfrak{J}^{C} \oplus \mathfrak{J}^{C} \oplus C \oplus C$. The simply connected compact Lie group $E_{7}$ is given by

$$
E_{7}=\left\{\alpha \in \operatorname{Iso}_{C}\left(\mathfrak{P}^{C}\right) \mid \alpha(P \times Q) \alpha^{-1}=\alpha P \times \alpha Q,\langle\alpha P, \alpha Q\rangle=\langle P, Q\rangle\right\} .
$$

Under the identification $\left(\mathfrak{P}_{\mathbf{C}}\right)^{C} \oplus\left(M(3, \mathbf{C})^{C} \oplus M(3, \mathbf{C})^{C}\right)$ with $\mathfrak{P}^{C}:((X, Y, \xi, \eta)$, $(M, N))=(X+M, Y+N, \xi, \eta), C$-linear transformations of $\gamma, \gamma^{\prime}, \gamma_{1}, \sigma$ and $\sigma^{\prime}$ of $\mathfrak{J}^{C}$ are extended to $C$-linear transformations of $\mathfrak{P}^{C}$ as

$$
\begin{aligned}
\gamma(X+M, Y+N, \xi, \eta) & =(X+\gamma M, Y+\gamma N, \xi, \eta), \\
\gamma^{\prime}(X+M, Y+N, \xi, \eta) & =\left(X+\gamma^{\prime} M, Y+\gamma^{\prime} N, \xi, \eta\right), \\
\gamma_{1}(X+M, Y+N, \xi, \eta) & =(\bar{X}+\bar{M}, \bar{Y}+\bar{N}, \xi, \eta), \\
\sigma(X+M, Y+N, \xi, \eta) & =(\sigma X+\sigma M, \sigma Y+\sigma N, \xi, \eta), \\
\gamma(X+M, Y+N, \xi, \eta) & =\left(\sigma^{\prime} X+\sigma^{\prime} M, \sigma^{\prime} Y+\sigma^{\prime} N, \xi, \eta\right),
\end{aligned}
$$

where $\gamma M=\operatorname{diag}(1,-1,-1) M, \gamma^{\prime} M=\operatorname{diag}(-1,-1,1) M, \sigma M=M \operatorname{diag}(1,-1,-1)$ and $\sigma^{\prime} M=M \operatorname{diag}(-1,-1,1)$.

Moreover we define a $C$-linear transformation $\iota$ of $\mathfrak{P}^{C}$ by

$$
\iota(X+M, Y+N, \xi, \eta)=(-i X-i M, i Y+i N,-i \xi, i \eta) .
$$


The group $\mathbf{Z}_{2}=\left\{1, \gamma_{1}\right\}$ acts the group $U(1) \times U(1) \times S U(6)$ by

$$
\gamma_{1}(p, q, A)=\left(\bar{p}, \bar{q}, \overline{\left.\left(\operatorname{Ad} J_{3}\right) A\right)}, \quad J_{3}=\left(\begin{array}{cc}
0 & E \\
-E & 0
\end{array}\right) .\right.
$$

Hence the group $\mathbf{Z}_{2}=\left\{1, \gamma_{1}\right\}$ acts naturally on the group $(U(1) \times U(1) \times S U(6)) / \mathbf{Z}_{3}$.

Let $(U(1) \times U(1) \times S U(6)) \cdot \mathbf{Z}_{2}$ be the semi-direct product of those groups under this action.

Proposition 4.1. $\left(E_{7}\right)^{\gamma, \gamma^{\prime}} \cong\left((U(1) \times U(1) \times S U(6)) / \mathbf{Z}_{3}\right) \cdot \mathbf{Z}_{2}, \mathbf{Z}_{3}=\{(1,1, E)$, $\left.\left(\omega_{1}, \omega_{1}, \omega_{1} E\right),\left(\omega_{1}^{2}, \omega_{1}^{2}, \omega_{1}^{2} E\right)\right\}$.

PRoOF. We define a mapping $\varphi_{7}:(U(1) \times U(1) \times S U(6)) \cdot \mathbf{Z}_{2} \rightarrow\left(E_{7}\right)^{\gamma, \gamma^{\prime}}$ by

$$
\begin{aligned}
\varphi_{7}((p, q, A), 1) P & =f^{-1}((D(p, q), A)(f P)), \\
\varphi_{7}\left((p, q, A), \gamma_{1}\right) P & =f^{-1}\left((D(p, q), A)\left(f \gamma_{1} P\right)\right), \quad P \in \mathfrak{P}^{C} .
\end{aligned}
$$

Here $D(p, q)=\operatorname{diag}(p, q, \overline{p q}) \in S U(3)$ and the mapping $f$ is defined in [9], Section 2.4. Then $\varphi_{7}$ induces the required isomorphism (see [5] for details).

LEMMA 4.2. The mapping $\varphi_{7}:(U(1) \times U(1) \times S U(6)) \cdot \mathbf{Z}_{2} \rightarrow\left(E_{7}\right)^{\gamma, \gamma^{\prime}}$ satisfies

$$
\sigma=\varphi_{7}\left(\left(1,1, F_{1,-1}\right), 1\right), \quad \sigma^{\prime}=\varphi_{7}\left(\left(1,1, F_{-1,1}\right), 1\right),
$$

where $F_{1,-1}=\operatorname{diag}(1,-1,-1,1,-1,-1), F_{-1,1}=\operatorname{diag}(-1,-1,1,-1,-1,1) \in S U(6)$.

The group $\mathbf{Z}_{2}=\left\{1, \gamma_{1}\right\}$ acts on the group $U(1)^{\times 7}$ by

$$
\gamma_{1}\left(p, q, a_{1}, a_{2}, a_{3}, a_{4}, a_{5}\right)=\left(\bar{p}, \bar{q}, \bar{a}_{4}, \bar{a}_{5}, \bar{a}_{1}, \bar{a}_{2}, \bar{a}_{3}\right) .
$$

Let $\left(U(1)^{\times 7}\right) \cdot \mathbf{Z}_{2}$ be the semi-direct product of those groups under this action.

Now, we determine the structures of the group $\left(E_{7}\right)^{\gamma, \gamma^{\prime}, \sigma, \sigma^{\prime}, \iota}=\left(\left(E_{7}\right)^{\gamma, \gamma^{\prime}}\right)^{\sigma, \sigma^{\prime}, \iota}$.

THEOREM 4.3. $\left(\left(E_{7}\right)^{\gamma, \gamma^{\prime}, \sigma, \sigma^{\prime}, l}\right)_{0} \cong U(1)^{\times 7}$.

Proof. For $\alpha \in\left(E_{7}\right)^{\gamma, \gamma^{\prime}, \sigma, \sigma^{\prime}, \iota} \subset\left(E_{7}\right)^{\gamma, \gamma^{\prime}}$, there exist $p, q \in U(1)$ and $A \in S U(6)$ such that $\alpha=\varphi_{7}((p, q, A), 1)$ or $\alpha=\varphi_{7}\left((p, q, A), \gamma_{1}\right)$ (Proposition 4.1). For the case of $\alpha=\varphi_{7}((p, q, A), 1)$, by combining the conditions $\sigma \alpha \sigma=\alpha, \sigma^{\prime} \alpha \sigma^{\prime}=\alpha$ and $\iota \alpha \iota^{-1}=\alpha$ with Lemma 4.2, we have

$\varphi_{7}\left(\left(p, q, F_{1,-1} A F_{1,-1}\right), 1\right)=\varphi_{7}((p, q, A), 1), \varphi_{7}\left(\left(p, q, F_{-1,1} A F_{-1,1}\right), 1\right)=\varphi_{7}((p, q, A), 1)$. and

$$
\varphi_{7}\left(\left(p, q, F_{e_{1}} A F_{e_{1}}^{-1}\right), 1\right)=\varphi_{7}((p, q, A), 1) .
$$

Hence
(i) $F_{1,-1} A F_{1,-1}=A$
(ii) $\left\{\begin{array}{l}p=\omega_{1} p \\ q=\omega_{1} q \\ F_{1,-1} A F_{1,-1}=\omega_{1} A,\end{array}\right.$
(iii) $\left\{\begin{array}{l}p=\omega_{1}^{2} p \\ q=\omega_{1}^{2} q \\ F_{1,-1} A F_{1,-1}=\omega_{1}^{2} A,\end{array}\right.$ 
(iv) $F_{-1,1} A F_{-1,1}=A, \quad(\mathrm{v})\left\{\begin{array}{l}p=\omega_{1} p \\ q=\omega_{1} q \\ F_{-1,1} A F_{-1,1}=\omega_{1} A,\end{array} \quad\right.$ (vi) $\left\{\begin{array}{l}p=\omega_{1}^{2} p \\ q=\omega_{1}^{2} q \\ F_{-1,1} A F_{-1,1}=\omega_{1}^{2} A .\end{array}\right.$

and

$$
\text { (vii) } F_{e_{1}} A F_{e_{1}}{ }^{-1}=A, \quad \text { (viii) }\left\{\begin{array} { l } 
{ p = \omega _ { 1 } p } \\
{ q = \omega _ { 1 } q } \\
{ F _ { e _ { 1 } } A F _ { e _ { 1 } } { } ^ { - 1 } = \omega _ { 1 } A , }
\end{array} \quad \text { (ix) } \left\{\begin{array}{l}
p=\omega_{1}^{2} p \\
q=\omega_{1}^{2} q \\
F_{e_{1}} A F_{e_{1}}{ }^{-1}=\omega_{1}^{2} A .
\end{array}\right.\right.
$$

We can eliminate the case (ii), (iii), (v), (vi), (viii) or (ix) because $p \neq 0$ or $q \neq 0$. Thus we have $p, q \in U(1)$ and $A \in S\left(U(1)^{\times 6}\right)$. Since the mapping $U(1)^{\times 5} \rightarrow S\left(U(1)^{\times 6}\right)$,

$$
h\left(a_{1}, a_{2}, a_{3}, a_{4}, a_{5}\right)=\left(a_{1}, a_{2}, a_{3}, a_{4}, a_{5}, \overline{a_{1} a_{2} a_{3} a_{4} a_{5}}\right)
$$

is an isomorphism, the group satisfying with the conditions of case (i), (iv) and (vii) is $\left(U(1)^{\times 7}\right) / \mathbf{Z}_{3}$. For the case of $\alpha=\varphi_{7}\left((p, q, A), \gamma_{1}\right)$, from $\varphi_{7}\left((p, q, A), \gamma_{1}\right)=\varphi_{7}((p, q, A)$, 1) $\gamma_{1}, \varphi_{7}\left(\left(1,1, F_{1,-1}\right), 1\right) \gamma_{1}=\gamma_{1} \varphi_{7}\left(\left(1,1, F_{1,-1}\right), 1\right), \varphi_{7}\left(\left(1,1, F_{-1,1}\right), 1\right) \gamma_{1}=\gamma_{1} \varphi_{7}((1$, $\left.\left.1, F_{-1,1}\right), 1\right)$, and $\varphi_{7}\left(\left(1,1, F_{e_{1}}\right), 1\right) \gamma_{1}=\gamma_{1} \varphi_{7}\left(\left(1,1, F_{e_{1}}\right), 1\right)$, this case is in the same situation as above. Thus we have $\left(E_{7}\right)^{\gamma, \gamma^{\prime}, \sigma, \sigma^{\prime}} \cong\left(\left(U(1)^{\times 7}\right) / \mathbf{Z}_{3}\right) \cdot \mathbf{Z}_{2}, \mathbf{Z}_{3}=\left\{(1)^{\times 7},\left(w_{1}\right)^{\times 7},\left(w_{1}^{2}\right)^{\times 7}\right\}$. The group $\left(U(1)^{\times 7}\right) / \mathbf{Z}_{3}$ is naturally isomorphic to the torus $U(1)^{\times 7}$, hence we obtain $\left(E_{7}\right)^{\gamma, \gamma^{\prime}, \sigma, \sigma^{\prime}, \iota} \cong\left(U(1)^{\times 7}\right) \cdot \mathbf{Z}_{2}$. Therefore we have the required isomorphism of the theorem.

\section{References}

[1] M. Berger, Les espaces symétriques non compacts, Ann. Sci. Ecole Norm. Sup. 74 (1957), 85-177.

[2] T. MiYashitA, Fixed points subgroups $G^{\sigma, \gamma}$ by two involutive automorphisms $\sigma, \gamma$ of compact exceptional Lie groups $G=F_{4}, E_{6}$ and $E_{7}$, Tsukuba J. Math. 27 (2003), 199-215.

[ 3 ] T. MiYAshita and I. Yokota, 2-graded decompositions of exceptional Lie algebra $\mathfrak{g}$ and group realizations of $\mathfrak{g}_{e v}, \mathfrak{g}_{0}$, Part III, $G=E_{8}$, Japanese J. Math. 26 (2000), 31-51.

[4] T. Miyashita and I. Yokota, Fixed points subgroups $G^{\sigma, \sigma^{\prime}}$ by two involutive automorphisms $\sigma, \sigma^{\prime}$ of compact exceptional Lie groups $G=F_{4}, E_{6}$ and $E_{7}$, Math. J. Toyama Univ. 24 (2001), 135-149.

[ 5] T. MiYashita and I. YokотA, Fixed points subgroups $G^{\gamma, \gamma^{\prime}}$ by two involutive automorphisms $\gamma, \gamma^{\prime}$ of compact exceptional Lie groups $G=G_{2}, F_{4}, E_{6}$ and $E_{7}$, Yokohama Math. J. 53 (2006), 9-38.

[6] T. MiYashita and I. YoкотA, 3-graded decompositions of exceptional Lie algebra $\mathfrak{g}$ and group realizations of $\mathfrak{g}_{e v}, \mathfrak{g}_{0}$ and $\mathfrak{g}_{e d}$, Part II, $G=E_{7}$, Part II, Case 1, J. Math. Kyoto Univ. 46-2 (2006), 383-413.

[ 7 ] T. Miyashita and I. Yokota, 3-graded decompositions of exceptional Lie algebra $\mathfrak{g}$ and group realizations of $\mathfrak{g}_{e v}, \mathfrak{g}_{0}$ and $\mathfrak{g}_{e d}$, Part II, $G=E_{7}$, Part II, Case 2, 3 and 4, J. Math. Kyoto Univ. 46-4 (2006), 805-832.

[ 8 ] I. YoкотA, Realization of involutive automorphisms $\sigma$ and $G^{\sigma}$ of exceptional linear Lie groups $G$, Part I, $G=G_{2}, F_{4}$, and $E_{6}$, Tsukuba J. Math. 4 (1990), 185-223.

[9] I. YoкотA, Realization of involutive automorphisms $\sigma$ and $G^{\sigma}$ of exceptional linear Lie groups $G$, Part II, $G=E_{7}$, Tsukuba J. Math. 4 (1990), 378-404.

[10] I. YокотA, 2-graded decompositions of exceptional Lie algebra $\mathfrak{g}$ and group realizations of $\mathfrak{g}_{\text {ev }}, \mathfrak{g}_{0}$, Part I, $G=G_{2}, F_{4}, E_{6}$, Japanese J. Math. 24 (1998), 257-296. 
[11] I. YокотA, 2-graded decompositions of exceptional Lie algebras $\mathfrak{g}$ and group realizations of $\mathfrak{g}_{e v}, \mathfrak{g}_{0}$, Part II, $G=E_{7}$, Japanese J. Math. 25 (1999), 155-179.

[12] I. YокотA, 3-graded decompositions of exceptional Lie algebra $\mathfrak{g}$ and group realizations of $\mathfrak{g}_{e v}, \mathfrak{g}_{0}$ and $\mathfrak{g}_{e d}$, Part II, $G=G_{2}, F_{4}, E_{6}$, Part I, J. Math. Kyoto Univ. 41-3 (2001), 449-474.

Present Address:

TOSHIKAZU MIYASHITA

KOMORO HIGH SCHOOL,

NAGANO, 384-0801 JAPAN.

e-mail: anarchybin@gmail.com 